\title{
ZINCO TOTAL E DISPONÍVEL EM AMOSTRAS DE PERFIS DE SOLOS DO ESTADO DE SÃO PAULO $\left({ }^{1}\right)$
}

\author{
GUSTAVO SOUZA VALLADARES $\left({ }^{2 *}\right)$; GLÁUCIA CECÍLIA GABRIELLI DOS SANTOS $\left({ }^{3}\right)$; \\ CLEIDE APARECIDA DE ABREU $\left({ }^{4}\right)$; OTÁVIO ANTÔNIO DE CAMARGO $\left({ }^{4}\right)$; JORGE PAZ FERRERO $\left({ }^{5}\right)$
}

\begin{abstract}
RESUMO
Em amostras dos horizontes A e B de vinte e oito perfis de unidades de solos representativas do Estado de São Paulo fez-se a determinação de zinco usando os extratores DTPA pH 7,3 (ZnDTPA); Mehlich-1 (ZnM1);

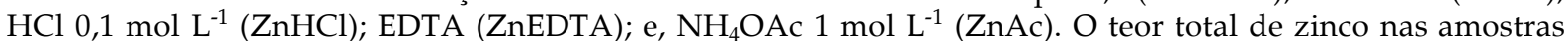
de solo estava alto, entretanto, notou-se somente uma pequena fração na forma disponível. O zinco total foi mais influenciado pelo teor de óxido de ferro $(\mathrm{r}=0,75)$. Para os teores considerados biodisponíveis, o $\mathrm{pH}$ foi o componente mais importante com coeficientes de correlação que variaram de 0,52 (ZnDTPA) a 0,66 (ZnAc). A análise dos componentes principais (PCA) revelou que os dois primeiros componentes principais explicaram $66 \%$ da variação, mostrando a associação entre os seguintes atributos dos solos: silte, pH, cálcio, magnésio, valor T, sílica, óxido de ferro, ZnT, ZnDTPA, ZnM1, ZnHCl e ZnEDTA, em que havia valores superiores a 0,63 no F1. No F2 ficou demonstrada a associação entre os teores de argila, areia fina e óxido de alumínio. Foi observada ambiguidade nos F1 e F2 para a areia grossa. Concluiu-se que: a maioria das amostras de solo apresenta potencial para o aparecimento de deficiência de zinco em curto e médio prazos e a PCA foi eficiente no agrupamento de solos originários de mesmo material de origem, indicando que as formas de Zn analisadas são influenciadas positivamente pelos teores de silte, $\mathrm{Ca}, \mathrm{Mg}$, sílica e $\mathrm{Fe}_{2} \mathrm{O}_{3}$, pH e valor T.
\end{abstract}

Palavras-chave: análise de solo, micronutriente, extratores químicos, análise multivariada, componente principal.

\section{ABSTRACT \\ TOTAL AND AVAILABLE ZINC IN SOIL PROFILES FROM SÃO PAULO STATE, BRAZIL}

Total and available zinc were analized in samples of A and/or B horizons of twenty eight representative soil profiles of the State of São Paulo, Brazil. Amounts of Zn extracted by DTPA pH 7.3 (Zn DTPA), Mehlich-1 (Zn M1), $\mathrm{HCl} 0.1$ mol. $\mathrm{L}^{-1}(\mathrm{Zn} \mathrm{HCl})$, EDTA (Zn-EDTA), and $\mathrm{NH}_{4} \mathrm{OAc}(\mathrm{Zn} \mathrm{Ac})$ were assessed. Amounts of total $\mathrm{Zn}(\mathrm{Zn} \mathrm{T})$ in all samples was high, but just a small fraction of it is available. Total $\mathrm{Zn}$ correlated well with iron oxide content $(\mathrm{r}=0.75)$. In terms of bioavailable amounts the $\mathrm{pH}$ was the most important component, showing correlation coefficients varying from 0.52 for $\mathrm{Zn}$ DTPA to 0.66 for $\mathrm{Zn}$ Ac. The two first axes of Principal Component Analysis (PCA) explained $66 \%$ of the variation, and showed association among the following soil attributes: silt, $\mathrm{pH}$, calcium, magnesium, CEC-value, silica, iron oxides, T-ZN, DTPA-Zn, M1-Zn, HCL-Zn and EDTA-Zn, that presented correlation values higher values than 0.63 with the main axis (FL). The second axis (F2) showed an association among clay content, fine sand, and aluminum oxide. An ambiguity was clear in F1 and F2 to coarse sand. It is concluded that: most samples have potential to show up zinc deficiency in the short and medium term; PCA was efficient in grouping soils from the same parent material, indicating that the $\mathrm{Zn}$ analyzed in this study is positively influenced by the silt, $\mathrm{Ca}, \mathrm{Mg}$, silica, and iron oxide contents, $\mathrm{pH}$, and CEC-value.

Key words: soil analysis, micronutrients, chemical extratants, multivariate analysis, principal component.

${ }^{1}$ ) Recebido para publicação em 6 de novembro de 2008 e aceito em 14 de julho de 2009.

$\left(^{2}\right)$ Universidade Federal do Ceará, Centro de Ciências Agrárias, Departamento de Ciências do Solo, Av. Mister Hull, 2977, Campus do Pici, bloco 807, Caixa Postal 12.168, 60021-970 Fortaleza (CE). E-mail: valladares@ufc.br (*) Autor correspondente.

$\left({ }^{3}\right)$ Aluna de pós-doutorado Instituto Agronômico, Av. Barão de Itapura, 1481, Caixa Postal 28, 13012-970 Campinas (SP). E-mail: gcgsantos@gmail.com.

$\left({ }^{4}\right)$ Instituto Agronômico, Av. Barão de Itapura, 1481, Caixa Postal 28, 13012-970 Campinas (SP). E-mail: cleide@iac.sp.gov.br, ocamargo@iac.sp.gov.br.

$\left(^{5}\right)$ Pesquisador com contrato pós doutorado, Facultad Ciências, Universidad de La Coruna, A Zapateira, 15071, A Coruña, Espanha. E-mail: jpaz@udc.es. 


\section{INTRODUÇÃO}

O zinco pode causar sérios distúrbios na nutrição vegetal, decorrentes da deficiência causada nos solos por sua baixa disponibilidade, da sua lixiviação nos solos arenosos com baixos teores de matéria orgânica e nos com pequena reserva de zinco total, e, em virtude de aplicação de calcário e adubos fosfatados em excesso.

A disponibilidade de zinco refere-se ao seu teor no solo resultante da inter-relação dos fatores intensidade, quantidade e poder tampão do solo durante um ciclo da planta, podendo ser avaliados por meio de extratores químicos.

Os extratores químicos podem ser classificados em soluções salinas, ácidas, quelantes, oxidantes/redutoras e mistas. Abreu et al. (2007) revisando os vários trabalhos de pesquisa sobre seleção de extratores de zinco em solos no Brasil, comentaram que não existem diferenças entre os métodos $\mathrm{HCl}$, Mehlich-1, Mehlich-3, DTPA e EDTA, quando se compara o coeficiente de correlação entre Zn-planta e Zn-solo. Segundo esses autores não é possível concluir qual é o melhor extrator, uma vez que os coeficientes de correlação estão muito próximos uns dos outros.

ORTIz et al. (2007) observaram valores de correlação de 0,93 para o Mehlich-1 e DTPA, concluindo que não existem diferenças significativas entre eles. No Brasil existem três métodos oficiais para a determinação de zinco, o DTPA pH 7,3 empregado no Estado de São Paulo; o ácido clorídrico usado no Rio Grande do Sul e Santa Catarina; e, o Mehlich-1 adotado em vários outros Estados como Minas Gerais, Espírito Santo e Goiás.

Embora, as diferenças nas eficiências dos métodos em determinar a disponibilidade de zinco em solos sejam pequenas, GALRÃO (1996) verificou que o Mehlich-1 foi mais eficiente em solos mais ácidos e o DTPA em solos mais alcalinos. NAScimento et al. (2002) comentaram que o extrator DTPA foi o de maior sensibilidade à mudança de $\mathrm{pH}$ do solo e que a solução extratora Mehlich-1, um extrator ácido, é a menos indicada para avaliar a disponibilidade de Zn, pois extrai formas pouco disponíveis às plantas ligadas a sítios de adsorção específica, como Zn ligado a hidróxidos e carbonatos. Portanto, ao que tudo indica, a interpretação do teor de zinco dos solos, extraído pelos vários métodos químicos, pode ser melhorada pela inclusão nos modelos matemáticos de alguns atributos do solo, principalmente do $\mathrm{pH}$.

A grande quantidade de informações químicas que podem ser obtidas com as análises de solo, remete à necessidade de se complementar a descrição estatística usando a análise simultânea de todas as amostras e variáveis. Os métodos estatísticos multivariados consideram as amostras e as variáveis em seu conjunto, permitindo extrair informações complementares que a análise univariada não consegue evidenciar (Moura et al., 2006).

A análise dos componentes principais (PCA) é uma técnica de análise multivariada baseada nas combinações lineares das variáveis originais. Os primeiros componentes principais explicam a maior parte da variância total contida no conjunto de dados e podem ser usados para representá-los (Moura et al., 2006). O método fornece as ferramentas adequadas para identificar as variáveis mais importantes no espaço das componentes principais (Moita Neto e Moita, 1998). Diversos trabalhos têm empregado a análise PCA para agrupar solos ou identificar a origem de metais pesados em solos e distinguir a contribuição natural e antrópica, destacando-se os trabalhos de FAcCHINELLI et al. (2001), BoruvKa et al. (2005), Moura et al. (2006) e Valladares et al. $(2008 ; 2009)$.

O objetivo deste trabalho foi avaliar o teor de zinco em vários perfis de solos do Estado de São Paulo e a influência de alguns atributos do solo nos teores de zinco, extraído por diferentes métodos, usando correlação linear de Pearson e a análise dos componentes principais.

\section{MATERIAL E MÉTODOS}

Amostras de horizontes superficiais e/ou subsuperficiais de 28 perfis de unidades de solos representativos do Estado de São Paulo (Figura 1), caracterizadas com maior detalhe no trabalho de VALADARES (1972), foram analisadas para a determinação do zinco. Destes, oito perfis eram originários de rochas básicas sendo dois perfis de Nitossolo Vermelho, quatro perfis de Latossolos Vermelhos férricos e dois perfis de Neossolos Litólicos; dois perfis originários de sedimentos modernos com influência de rochas básicas classificados como Latossolos Vermelhos; quatro perfis originários de sedimentos modernos classificados como Latossolos Vermelhos, sendo dois com horizonte A húmico; dois perfis originários de gnaisses classificados como Argissolos VermelhoAmarelos; quatro perfis originários de sedimentos do arenito Bauru, sendo quatro Argissolos VermelhoAmarelos; seis perfis originários do arenito Botucatu, sendo dois de Latossolos Vermelho-Amarelos, dois de Latossolos Vermelhos e dois de Neossolos Regolíticos; e dois perfis originários de sedimentos aluviais e coluviais classificados como Gleissolos. 


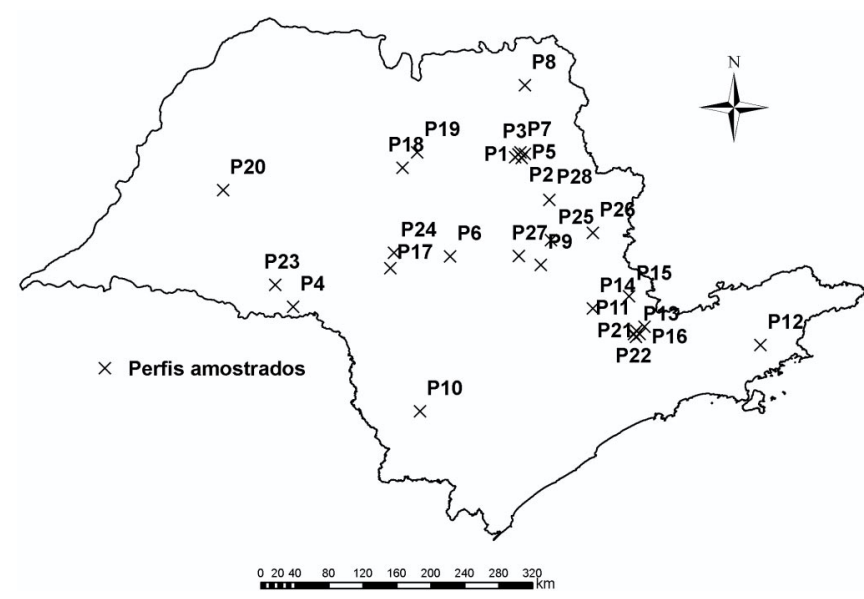

Figura 1. Localização dos perfis das amostras de solo no Estado de São Paulo.

As 57 amostras de solos foram submetidas à extração de zinco usando os seguintes métodos, com exceção do acetato de amônio que foi utilizado somente nas amostras coletadas na camada superficial:

a) ácido perclórico, fluorídrico e clorídrico (ZnT), segundo VALADAREs (1972)- $5 \mathrm{~g}$ de terra $+6 \mathrm{ml}$ de $\mathrm{HF}+1 \mathrm{ml} \mathrm{de} \mathrm{HCLO}_{4}$ concentrados $+5 \mathrm{ml} \mathrm{HCl} 5$ mol L ${ }^{-1}$; banho de areia a $200{ }^{0} \mathrm{C}$.

b) DTPA a pH 7,3 - (ZnDTPA), conforme LiNDSAy E NoRVEll $(1978)-10 \mathrm{~cm}^{3}$ de terra $+20 \mathrm{ml}$ de solução de ácido dietilenotriaminopentaacético 0,005M + trietanolamina 0,1 M + cloreto de cálcio 0,01 mol L ${ }^{-1}$ a pH 7,3; agitação por 2 horas.

c) Mehlich-1 - (ZnM1), descrito por Cox (1968) - $5 \mathrm{~cm}^{3}$ de TFSA + $20 \mathrm{ml}$ da solução de $\mathrm{HCl} \mathrm{0,05} \mathrm{mol}$ $\mathrm{L}^{-1}+\mathrm{H}_{2} \mathrm{SO}_{4} 0,025 \mathrm{~N}$; agitação por 15 minutos.

d) ácido clorídrico - $(\mathrm{ZnHCl})$, recomendado por WeAr e SOMMER (1948) - $5 \mathrm{~cm}^{3}$ de terra $+20 \mathrm{ml} \mathrm{de}$ solução de $\mathrm{HCl}$ 0,1 mol L ${ }^{-1}$; agitação por 30 minutos.

e) EDTA (ZnEDTA) segundo Viro (1955) - 5 $\mathrm{cm}^{3}$ de terra $+50 \mathrm{ml}$ de solução de ácido etilenodiaminoacético; agitação por 30 minutos.

f) acetato de amônio - (ZnAc), conforme AdAms (1965). Cinco $\mathrm{cm}^{3}$ de terra $+50 \mathrm{ml}$ de solução de $\mathrm{NH}_{4} \mathrm{OAc} 0,1 \mathrm{~mol} \mathrm{~L}^{-1}$; agitação por 30 minutos.

Os teores de $\mathrm{Zn}$ nos extratos de solo foram determinados por espectrometria de absorção atômica.

Os resultados foram submetidos à análise de correlação de Pearson, determinando-se a relação entre os teores de zinco (ZnT, ZnDTPA, ZnM1, ZnHCl, ZnEDTA e ZnAc) e alguns atributos dos solos (argila, silte, areia fina, areia grossa, $\mathrm{pH}$, cálcio, magnésio, potássio, valor T, óxido de ferro, óxido de alumínio, sílica, carbono, $\mathrm{Ki}$ ).
A comparação da capacidade extrativa dos diferentes métodos de zinco foi feita pelo uso da análise de regressão linear $\left(Y=b_{0}+b_{1} X\right)$, conforme recomendado por Miller e Miller (1993). A hipótese nula foi de que o coeficiente angular $\left(b_{1}\right)$ não fosse diferente de um (1), e o coeficiente linear ou intercepto $\left(b_{0}\right)$ não fosse diferente de zero (0). Ambas as hipóteses foram testadas pelo uso de intervalos de confiança a $95 \%$. Os resultados foram submetidos a análises de variância (teste F).

Os dados também foram submetidos a análise dos componentes principais (PCA) considerando os seguintes atributos dos solos: argila, silte, areia fina, areia grossa, $\mathrm{pH}$, cálcio, magnésio, potássio, valor $\mathrm{T}$, óxido de ferro, óxido de alumínio, sílica, carbono, $\mathrm{Ki}$, ZnT, ZnDTPA, ZnM1, ZnHCl, ZnEDTA e ZnAc. Os dados foram padronizados para média 0 e variância 1 e as análises foram realizadas na matriz de dados.

\section{RESULTADOS E DISCUSSÃO}

Dentre os métodos utilizados para avaliar o teor de zinco no solo, o $\mathrm{ZnT}$ foi o de maior capacidade extrativa seguido do $\mathrm{ZnHCl}, \mathrm{ZnM} 1, \mathrm{ZnEDTA}$ e ZnAc (Tabelas 1, 2 e 3). A explicação para este fato deve-se à interação do zinco com os componentes da fase sólida do solo, a natureza química dos extratores e a ordem de complexação dos elementos com os agentes quelantes. Somente uma pequena porcentagem do zinco total do solo está no "pool disponível" representado pelas formas solúvel em água, trocável e ligada à matéria orgânica (ABREu et al., 2002), uma vez que em solos tropicais existe a tendência do $\mathrm{Zn}$ a ligarse aos óxidos de Fe, Al e Mn (Machado e PAVAN, 1987).

Considerando somente os teores totais de zinco da camada superficial dos 28 perfis de solo, observa-se que em 50\% das amostras houve concentrações de zinco ( $\mathrm{ZnT}$ ) dentro do valor considerado como de referência de qualidade $(60 \mathrm{mg}$ $\mathrm{kg}^{-1}$ ), sugerido pela Cetesb (2008). O teor total de zinco das demais amostras, com exceção do Neossolo Litólico, ficou na faixa de prevenção, até $300 \mathrm{mg} \mathrm{kg}^{-1}$ (CETESB, 2008), bastante compreensível na medida da sua própria gênese e origem.

Comparando-se os teores de zinco extraídos com DTPA, método oficial para o Estado de São Paulo, com àqueles extraídos usando os métodos de Mehlich1, HCl, EDTA e acetato de amônio, verificase que para todos os métodos os coeficientes de determinação $\left(R^{2}\right)$ foram significativos ao nível de 5\% de probabilidade, indicando haver correspondência entre a capacidade extrativa dos métodos testados (Tabela 4). Contudo, para o ZnT e ZnAc os coeficientes de determinação foram baixos (Tabela 4). 
Tabela 1. Teores de zinco extraídos em perfis de solo do Estado de São Paulo, originários de rochas básicas ou de sedimentos com influências dessas rochas, usando vários extratores químicos

\begin{tabular}{|c|c|c|c|c|c|c|}
\hline & \multicolumn{6}{|c|}{ Zinco $\left(\mathrm{mg} \mathrm{kg}^{-1}\right)$} \\
\hline & Total & $\mathrm{NH}_{4} \mathrm{OAC}$ & DTPA & M-1 & $\mathrm{HCI}$ & EDTA \\
\hline \multirow{2}{*}{ Perfil Horizonte } & \multicolumn{6}{|c|}{ - Solos derivados de rochas básicas } \\
\hline & \multicolumn{6}{|c|}{ Neossolo Litólico } \\
\hline $\mathrm{P} 1 \mathrm{~A}^{(1)}$ & 315,30 & 0,40 & 2,20 & 3,60 & 6,20 & 4,70 \\
\hline P1 AC I & 282,70 & - & 0,40 & 3,60 & 1,80 & 1,00 \\
\hline \multirow[t]{2}{*}{ P2 A ${ }^{(1)}$} & 211,30 & 0,50 & 1,70 & 3,90 & 4,30 & 5,20 \\
\hline & \multicolumn{6}{|c|}{ Nitossolo Vermelho } \\
\hline P3 A1.1 (1) & 260,00 & 0,50 & 3,80 & 9,40 & 8,00 & 8,50 \\
\hline P3 B23 & 159,70 & - & 0,20 & 0,50 & 0,70 & 0,30 \\
\hline P4 Ap ${ }^{(1)}$ & 161,30 & 0,50 & 2,80 & 4,30 & 7,30 & 5,80 \\
\hline \multirow[t]{2}{*}{ P4 B22 } & 130,00 & - & 0,20 & 0,60 & 0,40 & 0,20 \\
\hline & \multicolumn{6}{|c|}{ Latossolo Vermelho férrico } \\
\hline P5 A1 (1) & 209,70 & 0,20 & 1,00 & 2,20 & 2,70 & 2,10 \\
\hline P5 B22' & 217,70 & - & 0,10 & 0,40 & 0,60 & 0,30 \\
\hline P6 A1 ${ }^{(1)}$ & 148,70 & 0,20 & 1,10 & 2,30 & 2,30 & 1,60 \\
\hline P6 B22 & 138,70 & - & 0,10 & 0,30 & 0,40 & 0,20 \\
\hline P7 Ap ${ }^{\prime(1)}$ & 87,00 & 0,20 & 0,30 & 0,80 & 0,70 & 0,30 \\
\hline P7 B22 & 90,00 & - & 0,10 & 0,60 & 0,30 & 0,20 \\
\hline P8 A1 (1) & 180,00 & 0,20 & 0,50 & 1,20 & 0,90 & 0,60 \\
\hline P8 B22‘ & 180,00 & & 0,10 & 0,20 & 0,20 & 0,20 \\
\hline
\end{tabular}

Solos derivados de sedimentos modernos com influência de rochas básicas

Latossolo Vermelho

\begin{tabular}{lcccccc} 
P9 A1 ${ }^{(1)}$ & 60,00 & 0,20 & 0,40 & 1,20 & 0,80 & 0,60 \\
P9 B22 & 54,00 & - & 0,10 & 0,40 & 0,10 & 0,10 \\
P10 A1 ${ }^{(1)}$ & 71,00 & 0,20 & 3,40 & 5,70 & 9,50 & 7,00 \\
P10 B21 & 59,00 & - & 0,10 & 0,50 & 0,50 & 0,10 \\
\hline
\end{tabular}

$\left({ }^{1}\right)$ Os símbolos indicam horizontes superficiais.

O DTPA extraiu menos zinco que os demais métodos, com exceção do acetato de amônio, uma vez que o coeficiente angular das retas para o $\mathrm{ZnT}, \mathrm{Zn}$ M1 e Zn EDTA foram superiores a 1 (Tabela 4). As soluções diluídas de ácidos fortes removem os metais da solução do solo, dos sítios de troca e parte daqueles complexados ou adsorvidos enquanto as soluções quelantes extraem os metais lábeis, porém sem dissolver as formas não lábeis, e as soluções salinas extraem, preferencialmente, os metais dos sítios de troca iônica do solo (ABREu et al., 2007). Portanto, a maior extração do $\mathrm{HCl}$ e do Mehlich-1 deve-se, provavelmente, à solubilização de parte do $\mathrm{Zn}$ adsorvido aos óxidos, enquanto os outros extratores (EDTA, DTPA e Ac) não conseguiram fazê-lo.
Embora, os métodos químicos de extração e os critérios de partição dos resultados de análise de zinco em classes de teores variam em função da região, já existem tabelas de interpretação dos resultados da análise de solo, definidas por órgãos oficiais, existentes nos Estados do Rio Grande do Sul, de Santa Catarina, do Paraná, Espírito Santo, de São Paulo, Minas Gerais e para solos dos cerrados (ABREU et al., 2007).

Para o Estado de São Paulo, Abreu et al. (2005) sugerem os seguintes valores de interpretação para $\mathrm{Zn}$, extraídos com DTPA pH 7,3, em $\mathrm{mg} \mathrm{dm}^{-3}$ : baixo $(0-0,5)$, médio $(0,6-1,2)$, alto $(1,3-2,3)$, muito alto $(2,4-$ $15)$, toxidez $(>130)$. 
Tabela 2. Teores de zinco extraídos em perfis de solo do Estado de São Paulo, originários de sedimentos modernos ou do arenito Bauru, usando vários extratores químicos

\begin{tabular}{|c|c|c|c|c|c|c|}
\hline \multirow[b]{3}{*}{ P11 A11 ${ }^{(1)}$} & \multicolumn{6}{|c|}{ Solos derivados de sedimentos modernos } \\
\hline & \multicolumn{6}{|c|}{ Latossolo Vermelho } \\
\hline & 66,00 & 0,40 & 0,90 & 1,80 & 2,40 & 1,30 \\
\hline P11 B22 & 39,00 & - & 0,10 & 0,30 & 0,10 & 0,20 \\
\hline P12 A11 (1) & 33,00 & 0,20 & 0,40 & 0,80 & 0,80 & 0,20 \\
\hline P12 B22 & 36,00 & - & 0,10 & 0,30 & 0,10 & 0,10 \\
\hline P13 Ap ${ }^{(1)}$ & 44,00 & 0,10 & 0,30 & 0,60 & 0,70 & 0,20 \\
\hline P13 B2 & 53,00 & - & 0,10 & 0,20 & 0,20 & 0,10 \\
\hline P14 A11 (1) & 22,70 & 0,10 & 0,10 & 0,30 & 0,20 & 0,20 \\
\hline \multirow[t]{2}{*}{ P14 B22 } & 33,70 & - & 0,10 & 0,70 & 0,20 & 0,10 \\
\hline & \multicolumn{6}{|c|}{ Argissolo Vermelho-Amarelo } \\
\hline P15 A1 ${ }^{(1)}$ & 35,00 & 0,40 & 1,30 & 1,80 & 2,10 & 1,70 \\
\hline P15 B22 & 33,00 & - & 0,10 & 0,50 & 0,40 & 0,20 \\
\hline P16 Ap ${ }^{(1)}$ & 30,00 & 0,20 & 0,30 & 1,10 & 1,00 & 0,60 \\
\hline P16 B22 & 33,00 & - & 0,10 & 0,40 & 0,50 & 0,10 \\
\hline \multirow[t]{2}{*}{ P16 B3 } & 33,00 & - & 0,10 & 0,40 & 0,30 & 0,10 \\
\hline & \multicolumn{6}{|c|}{$\begin{array}{c}\text { Solos derivados de sedimento arenito Bauru } \\
\text { Argissolo Vermelho-Amarelo }\end{array}$} \\
\hline P17 Ap ${ }^{(1)}$ & 33,70 & 0,40 & 0,80 & 1,90 & 1,90 & 1,30 \\
\hline P17 B22 & 27,00 & - & 0,10 & 0,20 & 0,40 & 0,10 \\
\hline P18 A1 ${ }^{(1)}$ & 21,00 & 0,20 & 0,40 & 0,90 & 1,00 & 0,50 \\
\hline P18 B22 & 27,30 & - & 0,10 & 0,40 & 0,40 & 0,10 \\
\hline P19 Ap ${ }^{(1)}$ & 18,00 & 0,30 & 0,70 & 1,40 & 1,30 & 1,30 \\
\hline P19 B21 & 27,00 & - & 0,10 & 0,30 & 0,30 & 0,30 \\
\hline P20 A11 (1) & 16,50 & 0,30 & 0,10 & 0,20 & 1,20 & 0,40 \\
\hline P20 E3 & 23,50 & - & 0,10 & 0,20 & 0,40 & 0,30 \\
\hline
\end{tabular}

$\left({ }^{1}\right)$ símbolos em negrito indicam horizontes superficiais.

Baseando-se nestes valores, $57 \%$ das amostras do horizonte A tiveram valores baixos, 21\% dos valores foram considerados médios, $7 \%$ altos e $15 \%$ muito altos, nas duas classes mais altas a maioria dos solos eram derivados de rochas básicas (Tabela 1).

Para a extração de zinco das amostras de solos dos cerrados, adotou-se a solução de Mehlich-1 com os seguintes valores de interpretação, $\mathrm{em} \mathrm{m} \mathrm{dm}^{-3}$ : baixo $(0-1,0)$, médio $(1,1-1,6)$, alto $(>1,6)$ (GALRÃo et al., 2002). Das amostras dos horizontes superficiais, em $46 \%$ das amostras os valores baixos, $19 \%$ dos valores considerados médios, $35 \%$ altos, e nesta última classe a maioria dos solos eram derivados de rochas básicas (Tabela 1).

Nos Estados de Santa Catarina e Rio Grande do Sul os limites de interpretação para os teores de zinco extraído por $\mathrm{HCl}$, em $\mathrm{mg} \mathrm{dm}^{-3}$ são: baixo $(<0,2)$, médio $(0,2-0,5)$ e alto $(>0,5)$ (Comissão De Fertilidade Do Solo - RS/SC, 1994). O equivalente a $14 \%$ das amostras foi de baixos teores, $11 \%$ teores médios e $75 \%$ altos teores, a maioria de solos originários de rochas básicas.

Comparando os três métodos de análise no que se refere à distribuição de zinco em classes de teores, verificou-se que o DTPA e o Mehlich-1 comportamentos semelhantes entre si, mas diferentes do extrator $\mathrm{HCl}$. Para o DTPA e M-1, na maioria das amostras observaram-se teores de zinco referente à classe de teor baixo, seguindo-se das classes de teor alto e médio. Quando se utilizou o $\mathrm{HCl}$, a maioria das amostras estava na classe de interpretação alta, vindo mostrar, mais uma vez, que não se deve usar a tabela de interpretação de um método para interpretar os resultados de análise de zinco extraído por outro método. 
Tabela 3. Teores de zinco extraídos em perfis de solo do Estado de São Paulo, originários de sedimentos aluviais e coluviais ou do arenito Botucatu, usando vários extratores químicos

\begin{tabular}{|c|c|c|c|c|c|c|}
\hline \multirow[b]{3}{*}{ P21 Ap ${ }^{(1)}$} & \multicolumn{6}{|c|}{ Solos derivados de sedimentos aluviais e coluviais } \\
\hline & \multicolumn{6}{|c|}{ Gleissolo } \\
\hline & 53,00 & 0,40 & 0,40 & 0,80 & 1,60 & 0,90 \\
\hline P21 C2g & 66,00 & - & 0,10 & 0,60 & 0,30 & 0,10 \\
\hline P22 A1 (1) & 82,00 & 0,30 & 0,30 & 1,10 & 1,10 & 0,60 \\
\hline P22 C1g & 102,00 & - & 0,20 & 0,70 & 1,00 & 0,40 \\
\hline \multirow[t]{3}{*}{ P23 C3g } & 102,00 & - & 0,10 & 0,50 & 0,50 & 0,20 \\
\hline & \multicolumn{6}{|c|}{ Solos derivados de sedimento arenito Botucatu } \\
\hline & \multicolumn{6}{|c|}{ Latossolo Vermelho-Amarelo } \\
\hline P23 A1 ${ }^{(1)}$ & 16,70 & 0,10 & 0,10 & 0,20 & 0,20 & 0,10 \\
\hline P23 B22 & 16,70 & - & 0,10 & 0,10 & 0,10 & 0,10 \\
\hline P24 A1 (1) & 5,00 & 0,10 & 0,10 & 0,20 & 0,30 & 0,20 \\
\hline \multirow[t]{2}{*}{ P24 B22 } & 7,30 & - & 0,10 & 0,10 & 0,20 & 0,10 \\
\hline & \multicolumn{6}{|c|}{ Latossolo Vermelho } \\
\hline P25 Ap ${ }^{(1)}$ & 8,30 & 0,10 & 0,30 & 0,40 & 0,40 & 0,30 \\
\hline P25 B22 & 14,00 & - & 0,10 & 0,20 & 0,30 & 0,10 \\
\hline P26 A1 ${ }^{(1)}$ & 8,30 & 0,10 & 0,10 & 0,30 & 0,20 & 0,20 \\
\hline \multirow[t]{2}{*}{ P26 B22 } & 13,70 & - & 0,10 & 0,20 & 0,20 & 0,10 \\
\hline & \multicolumn{6}{|c|}{ Neossolo Regolítico } \\
\hline P27 Ap ${ }^{(1)}$ & 1,00 & 0,10 & 0,20 & 0,40 & 0,20 & 0,20 \\
\hline P27 A14 ${ }^{(1)}$ & 10,50 & - & 0,10 & 0,30 & 0,10 & 0,10 \\
\hline P28 A11 ${ }^{(1)}$ & 1,00 & 0,10 & 0,20 & 0,20 & 0,20 & 0,30 \\
\hline P28 C3 & 5,80 & - & 0,10 & 0,30 & 0,10 & 0,10 \\
\hline Média* & 73,9 & 0,3 & 0,5 & 1,1 & 1,2 & 0,9 \\
\hline Mínimo* & 1,0 & 0,1 & 0,1 & 0,1 & 0,1 & 0,1 \\
\hline Máximo* & 315,3 & 0,5 & 3,8 & 9,4 & 9,5 & 8,5 \\
\hline Desvio-Padrão* & 77,6 & 0,1 & 0,8 & 1,6 & 2,0 & 1,8 \\
\hline Coeficiente Variação* & * 105 & 55 & 165 & 146 & 161 & 192 \\
\hline Tamanho amostral* & 57 & 28 & 57 & 57 & 57 & 57 \\
\hline
\end{tabular}

$\left({ }^{1}\right)$ símbolos indicam horizontes superficiais.

*Estatística descritiva calculada de dados de todos os perfis.

Com base na análise de correlação linear de Pearson, verifica-se que de maneira geral, os atributos do solo que tiveram maior influência no teor de zinco extraído pelos diferentes métodos foram o $\mathrm{pH}$, silte, cálcio, magnésio e potássio, com coeficientes de correlação variando, respectivamente, de 0,52 a 0,73 , de 0,41 a $0,73,0,48$ a $0,75,0,55$ a 0,74 e 0,54 a 0,67 (Tabela 5). Os teores de $\mathrm{Fe}_{2} \mathrm{O}_{3}$, carbono orgânico e T também revelaram coeficientes de correlação significativos, contudo mais baixos (Tabela 5).

$\mathrm{O} \mathrm{ZnT}$ foi mais influenciado pelos teores de óxido de ferro e cálcio $(\mathrm{r}=0,75)$, seguindo-se do teor de silte e do valor de $\mathrm{pH}(\mathrm{r}=0,73)$ (Tabela 5). A associação do ZnT com óxidos e argilominerais também foi reportada por KPARMWANG et al. (1998) em solos da Nigéria.

Para os teores considerados biodisponíveis, o $\mathrm{pH}$ foi o mais importante com coeficientes de correlação que variaram de 0,52 (ZnDTPA) a 0,66 (ZnAc) (Tabela 5) . Contudo, não era esperada a correlação positiva entre teor de zinco e $\mathrm{pH}$, pois, conforme SHUMAN (1991) o fato de a disponibilidade de zinco diminuir com o aumento de $\mathrm{pH}$ é bastante conhecida na literatura, sendo provável que ocorresse também aqui se fossem estudadas as variações destes dois componentes para a mesma amostra de solo. 
Porém, nas amostras estudadas, em solos originários de rochas básicas constataram-se maiores valores de pH e de Zn, como já discutido anteriormente, o que tornou esta correlação positiva.

VALLADARES et al. (1997), trabalhando com 103 amostras de horizontes superficiais de solos do Estado do Rio de Janeiro verificou correlação positiva e significativa dos teores disponíveis de Zn extraídos com $\mathrm{HCl}$ e DTPA e os valores de $\mathrm{pH}$. Além do $\mathrm{pH}$, o silte e o teor de óxido de ferro e o carbono influenciaram na extração do zinco considerado biodisponível, sendo os coeficientes de correlação muito próximos uns dos outros (Tabela 5).

A associação das formas disponíveis de zinco (DTPA) com a matéria orgânica e pH do solo também foi reportada por Udo E FAGBAMI (1979). Entretanto, o mesmo não foi observado para o zinco extraído com HCl. Já KPARMWANG et al. (1998), em solos da Nigéria, observaram correlações positivas significativas entre ZnDTPA e silte e entre ZnDTPA e pH, em solos originários de basaltos e correlações negativas significativas entre ZnDTPA e matéria orgânica.

Tabela 4. Intervalo de confiança dos coeficientes angulares e lineares da regressão linear estimada entre os teores de zinco extraídos pelo DTPA $(\mathrm{X})$ com os extraídos por ácido forte (ZnT), Melich 1 (ZnM1), HCl, EDTA e acetato de amônio (ZnAc) (Y) em diferentes perfis de solos do Estado de São Paulo

\begin{tabular}{|c|c|c|c|c|c|c|c|c|c|}
\hline \multirow{2}{*}{$\mathrm{Y}$} & \multirow{2}{*}{$x$} & \multicolumn{3}{|c|}{ Coeficiente angular } & \multicolumn{3}{|c|}{ Coeficiente linear } & \multirow{2}{*}{$r^{2}$} & \multirow{2}{*}{$\mathrm{F}$} \\
\hline & & Mínimo & Médio & Máximo & Mínimo & Médio & Máximo & & \\
\hline $\mathrm{ZnT}$ & ZnDTPA & 26,63 & 49,19 & 71,75 & 29,03 & 50,04 & 71,04 & $0,26^{*}$ & $19,1^{*}$ \\
\hline ZnM1 & ZnDTPA & 1,72 & 1,90 & 2,09 & 0,01 & 0,18 & 0,35 & $0,89 *$ & $436,6^{*}$ \\
\hline $\mathrm{ZnHCl}$ & ZnDTPA & 2,31 & 2,44 & 2,58 & $-0,07$ & 0,05 & 0,18 & $0,96^{*}$ & $1314,4^{*}$ \\
\hline ZnEDTA & ZnDTPA & 2,06 & 2,17 & 2,28 & $-0,24$ & $-0,14$ & $-0,03$ & $0,96^{*}$ & $1583,3^{*}$ \\
\hline $\mathrm{ZnAc}$ & ZnDTPA & 0,04 & 0,08 & 0,13 & 0,12 & 0,18 & 0,23 & $0,39^{*}$ & $32,5^{*}$ \\
\hline
\end{tabular}

* significativo a $\mathrm{P}<0,05$

Tabela 5. Coeficientes de correlação entre o zinco extraído por diferentes métodos e alguns atributos dos solos do Estado de São Paulo

\begin{tabular}{|c|c|c|c|c|c|c|}
\hline Atributos & $\mathrm{ZnT}$ & ZnDTPA & ZnM1 & $\mathrm{ZnHCI}$ & ZnEDTA & $\mathrm{ZnAc}^{*}$ \\
\hline argila & $0.32^{*}$ & -0.01 & 0.01 & 0.02 & -0.01 & 0.20 \\
\hline silte & $0.73^{*}$ & $0.41^{*}$ & $0.48^{*}$ & $0.42^{*}$ & $0.42^{*}$ & $0.64^{*}$ \\
\hline areia fina & $-0.39^{*}$ & -0.13 & -0.15 & -0.14 & -0.11 & -0.24 \\
\hline areia grossa & $-0.54^{*}$ & -0.13 & -0.17 & -0.17 & -0.16 & -0.44 \\
\hline $\mathrm{pH}$ & $0.73^{*}$ & $0.52^{*}$ & $0.55^{*}$ & $0.59 *$ & $0.56^{*}$ & $0.66^{*}$ \\
\hline carbono & 0.20 & $0.31^{*}$ & $0.31^{*}$ & $0.32^{*}$ & $0.28^{*}$ & $0.39^{*}$ \\
\hline cálcio & $0.75^{*}$ & $0.48^{*}$ & $0.63^{*}$ & $0.53^{*}$ & $0.52^{*}$ & $0.60^{*}$ \\
\hline magnésio & $0.55^{*}$ & $0.71^{*}$ & $0.72^{*}$ & $0.71^{*}$ & $0.74^{*}$ & $0.55^{*}$ \\
\hline potássio & $0.60^{*}$ & $0.56^{*}$ & $0.54^{*}$ & $0.59^{*}$ & $0.60^{*}$ & $0.67^{*}$ \\
\hline Valor T & $0.50^{*}$ & $0.35^{*}$ & $0.42^{*}$ & $0.41^{*}$ & $0.35^{*}$ & $0.49^{*}$ \\
\hline $\mathrm{SiO} 2$ & $0.42^{*}$ & 0.12 & 0.20 & 0.17 & 0.14 & 0.41 \\
\hline AI203 & $0.41^{*}$ & 0.01 & 0.05 & 0.03 & 0.00 & 0.17 \\
\hline Fe203 & $0.75^{*}$ & $0.3^{*}$ & $0.38^{*}$ & $0.35^{*}$ & $0.36^{*}$ & 0.37 \\
\hline $\mathrm{Ki}$ & 0.23 & 0.17 & $0.29^{*}$ & 0.23 & 0.21 & $0.49^{*}$ \\
\hline ZnT & - & $0.50^{*}$ & $0.61^{*}$ & $0.53^{*}$ & $0.54^{*}$ & $0.57^{*}$ \\
\hline ZnDTPA & - & - & $0.94^{*}$ & $0.98^{*}$ & $0.98^{*}$ & $0.63^{*}$ \\
\hline ZnM1 & - & - & - & $0.92^{*}$ & $0.95^{*}$ & $0.63^{*}$ \\
\hline ZnHCI & - & - & - & - & $0.97^{*}$ & $0.62^{*}$ \\
\hline ZnEDTA & - & - & - & - & - & $0.65^{*}$ \\
\hline $\mathrm{ZnAc} c^{*}$ & - & - & - & - & - & - \\
\hline
\end{tabular}

* significativo a $\mathrm{p}<0,05$. 
A análise dos componentes principais (Tabela 6) revelou que os dois primeiros componentes principais (representados por F1 e F2) explicaram 66\% da variação (Tabela 6). Alguns trabalhos consideram os três primeiros componentes principais (FACCHINELLI et al., 2001; BorUvKa et al., 2005), que no presente trabalho explicaram $76 \%$.

Tabela 6. Eigenvalue e \% da variância pela análise dos componentes principais para as amostras dos solos estudadas

\begin{tabular}{lccc}
\hline & F1 & F2 & F3 \\
\hline Eingenvalue & 8,414 & 4,188 & 1,920 \\
\% Variância & 44,282 & 22,040 & 10,105 \\
\% Acumulada & 44,282 & 66,322 & 76,427 \\
\hline
\end{tabular}

Os resultados iniciais revelaram associação entre os seguintes atributos dos solos: silte, $\mathrm{pH}$, cálcio, magnésio, valor $\mathrm{T}$, sílica, óxido de ferro, $\mathrm{ZnT}$, ZnDTPA, ZnM1, ZnHCl, ZnEDTA, com valores superiores a 0,63 (Tabela 7) no F1. No F2 ficou demonstrada a associação entre os teores de argila, areia fina e óxido de alumínio. Foi observada ambigüidade nos F1 e F2 para a areia grossa. Em Ki os valores foram mais altos no F3 (Tabela 7).

Tabela 7. Fatores calculados pela análise dos componentes principais para as amostras estudadas com base nas matrizes dos teores dos elementos pesquisados

\begin{tabular}{lccc}
\hline Variáveis & $\mathrm{F} 1$ & $\mathrm{~F} 2$ & $\mathrm{~F} 3$ \\
\hline Argila & 0,449 & 0,814 & $-0,108$ \\
Silte & 0,794 & 0,299 & $-0,014$ \\
Areia fina & $-0,533$ & $-0,714$ & 0,079 \\
Areia grossa & $-0,595$ & $-0,520$ & 0,059 \\
$\mathrm{pH}$ & 0,763 & $-0,131$ & $-0,143$ \\
$\mathrm{C}$ & 0,493 & 0,212 & 0,473 \\
$\mathrm{Ca}$ & 0,680 & $-0,390$ & 0,317 \\
$\mathrm{Mg}$ & 0,757 & $-0,197$ & $-0,242$ \\
$\mathrm{~K}$ & 0,631 & $-0,417$ & 0,256 \\
$\mathrm{~T}$ & 0,703 & 0,154 & 0,593 \\
$\mathrm{SiO}_{2}$ & 0,632 & 0,550 & 0,405 \\
$\mathrm{AI} \mathrm{O}_{3}$ & 0,497 & 0,823 & $-0,080$ \\
$\mathrm{Fe}_{2} \mathrm{O}_{3}$ & 0,634 & 0,235 & $-0,453$ \\
$\mathrm{Ki}_{\mathrm{Zn} \mathrm{T}}$ & 0,280 & $-0,462$ & 0,709 \\
$\mathrm{Zn} \mathrm{DTPA}$ & 0,843 & $-0,006$ & $-0,073$ \\
$\mathrm{Zn} \mathrm{NC}$ & 0,742 & $-0,495$ & $-0,262$ \\
$\mathrm{Zn} \mathrm{HCI}$ & 0,789 & $-0,471$ & $-0,162$ \\
$\mathrm{Zn} \mathrm{EDT} \mathrm{A}$ & 0,773 & $-0,481$ & $-0,201$ \\
\hline
\end{tabular}

Pelo agrupamento das amostras de solo, observa-se que no terceiro quadrante, com valores para F1 e F2 negativos, estão posicionadas as amostras de solos originários dos arenitos Bauru e Botucatu, havendo uma nítida separação entre os dois materiais de origem (Figura 2). Estas amostras de solo são caracterizadas por uma textura arenosa ou média, com predomínio da fração areia fina, menores teores de carbono orgânico; valores de $\mathrm{pH}$ de moderados a altos (arenito Bauru) e entre baixos e moderados (arenito Botucatu); baixos teores de $\mathrm{Fe}_{2} \mathrm{O}_{3}$; e teores baixos de Zn quando comparados aos solos derivados dos demais materiais de origem.

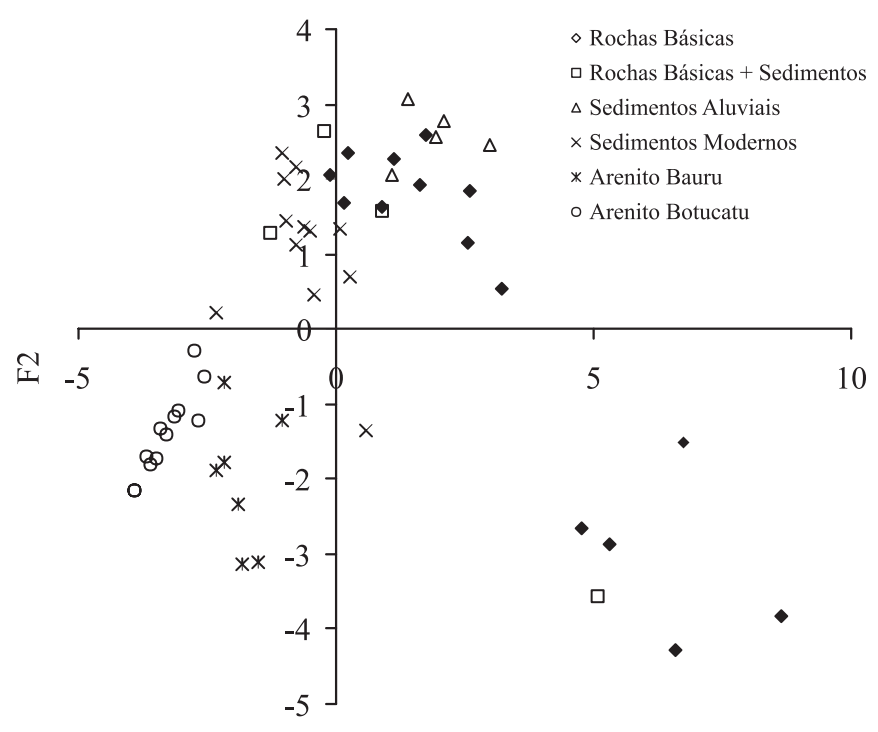

F1

Figura 2. Agrupamento das amostras de solo pela análise dos componentes principais.

As amostras de solos originárias de sedimentos modernos tiveram bom agrupamento, posicionando-se principalmente no segundo quadrante, isto é, com valores de F1 negativos e de F2 positivos para a maioria das amostras (Figura 2). Nessas amostras a textura situou-se entre média e argilosa, $\mathrm{pH}$ variando de 4,4 a 5,5, teores baixos e médios de $\mathrm{Fe}_{2} \mathrm{O}_{3}$, teores de $\mathrm{SiO}_{2}$ e Zn de médios a altos.

As amostras originárias de sedimentos aluviais posicionaram-se no primeiro quadrante (Figura 2), com bom agrupamento. Essas amostras são de textura argilosa, $\mathrm{pH}$ entre 4,4 a 5,5, baixos e médios teores de $\mathrm{Fe}_{2} \mathrm{O}_{3}$ e altos teores das formas de $\mathrm{Zn}$, porém inferiores aos valores verificados nos solos originários de basalto. 
Das quinze amostras originárias de rochas básicas, nove posicionaram-se no primeiro quadrante e uma no segundo, próxima das demais, com textura mais argilosa e de solos bastante intemperizados, e cinco no quarto quadrante com textura média e solos pouco intemperizados (Figura 2). As amostras do primeiro quadrante agruparam-se parcialmente com amostras de solos originários de sedimentos aluviais, em razão da semelhança nos atributos estudados. Quanto aos demais atributos, esses solos foram os de valores mais elevados de $\mathrm{pH}$, superiores a 5,5, e altos teores de $\mathrm{Fe}_{2} \mathrm{O}_{3}$ e de $\mathrm{SiO}_{2}$. Nestes solos foram verificados os mais elevados teores das formas de $\mathrm{Zn}$.

Os solos originários de rochas básicas com influência de sedimentos tiveram o pior agrupamento entre si, ficando uma das amostras no quarto quadrante, agrupada as amostras de solos originários de rochas básicas e com atributos semelhantes aos dela. Outras duas amostras posicionaram-se no segundo quadrante, próximas a amostras originárias de sedimentos modernos. Uma quarta amostra posicionou-se agrupada a outras amostras de solos originários de basalto, com propriedades semelhantes a esses solos. Estes agrupamentos fazem sentido uma vez que metade das amostras ficou mais próxima dos basaltos e metade dos sedimentos modernos, sendo todas semelhantes com esses dois grupos.

\section{CONCLUSÕES}

1. Os teores de zinco em solos representativos do Estado de São Paulo são relativamente altos, mas apenas uma pequena fração destes está disponível. Portanto, a maioria das amostras de solo tem potencial para o aparecimento de deficiência de zinco a curto e médio prazo.

2. As elevadas correlações entre os extratores DTPA, EDTA, $\mathrm{HCl}$ e Mehlich-1 indicam similaridade nas formas extraídas de Zn pelos extratores.

3. A PCA é eficiente no agrupamento de solos originários de mesmo material de origem e indica que as formas de $\mathrm{Zn}$ analisadas são influenciadas positivamente pelo $\mathrm{pH}$, teores de silte, $\mathrm{Ca}, \mathrm{Mg}$, sílica e $\mathrm{Fe}_{2} \mathrm{O}_{3}$, e valor $\mathrm{T}$.

\section{REFERÊNCIAS}

ABREU, C.A.; LOPES, A.S.; SANTOS, G.C.G. Micronutrientes. In: NOVAIS, R.F.N.; ALVAREZ V, V. H; BARROS, N. F.; FONTES, R.L.F.; CANTARUTTI, R.B. NEVES, J.C.L (Ed.). Fertilidade do Solo. Viçosa: Sociedade Brasileira de Ciência do Solo, 2007. p.645-736.
ABREU, C.A.; ABREU, M.F.; BERTON, R.S. Análise química de solo para metais pesados. In: ALVAREZ V, V. H; SCHAEFER, C. E. G. R.; BARROS, N. F.; MELLO, J. W. V. E COSTA, L. M. (Ed.). Tópicos em Ciência do Solo. Viçosa: Sociedade Brasileira de Ciência do Solo, 2002. p.645-692.

ABREU, C.A; RAIJ, B. van; ABREU, M.F.; Paz-Gonzalez, A. Routine soil testing to monitor heavy metals and boron in soils. Scientia Agrícola, v.62, p.564-571, 2005.

ADAMS, F. Manganese. In: BLACK, C.A. (Ed.). Methods of soil analysis. Madison: American Society Agronomy, 1965. Part 2, p.1011-1018.

BORUVKA, L.; VACEK, O.; JEHLICKA, J. Principal component analysis as a tool to indicate the origin of potentially toxic elements in soils. Geoderma, v.128, p.289-300, 2005.

CETESB - COMPANHIA DE TECNOLOGIA DE SANEAMENTO AMBIENTAL. Relação de áreas contaminadas no estado de São Paulo. http:/ /www.cetesb.sp.gov.br/Solo/ areas_contaminadas/areas.asp. Acesso em 8 agosto 2008.

COMISSÃO DE FERTILIDADE DO SOLO - RS/SC. Recomendações de adubação e de calagem para os Estados do Rio Grande do Sul e de Santa Catarina. 3.ed. Passo Fundo: SBCS - Núcleo Regional Sul, 1994. 224p.

COX, F.R. Development of a yield response prediction and manganese soil test interpretation for soybeans. Agronomy Journal, v.60, p.521-524, 1968.

FACCHINELLI, A.; SACCHI, E.; MALLEN, L. Multivariate statistical and GIS-based approach to identify heavy metal sources in soils. Environmental Pollution, v.114, p. 313-324, 2001.

GALRÃO, E.Z. Níveis críticos de zinco e avaliação de sua disponibilidade para o milho num Latossolo VermelhoEscuro, argiloso, fase cerrado. Revista Brasileira de Ciência do Solo, v.20, p.283-289, 1996.

GALRÃO, E.Z. Micronutrientes. In: SOUSA, D.M.G.; LOBATO, E. (Ed.) Cerrado - correção do solo e adubação. Planaltina, DF : Embrapa Cerrados, 2002. p.185-226.

KPARMWANG, T.;SEU, I.E.; CHUDE, V.O. Available and total forms of copper and zinc in basaltic soils of the Nigerian Savanna. Communications in Soil Science and Plant Analysis, v.29, p. 2235-224, 1998.

LINDSAY, W.L.; NORVELL, W.A. Development of DTPA soil test for zinc, iron, manganese and copper. Soil Science Society of America Journal, v.42, p.421-428, 1978.

MACHADO, P.L.O.A.; PAVAN, M.A. Adsorção de zinco por alguns solos do Paraná. Revista brasileira de Ciência do Solo, v.11, p.253-256, 1987.

MILLER, J.C. MILLER, J.N. Statistics for analytical chemistry. 3.ed. New York: Ellis Horwood, 1993. 256p

MOITA NETO, J.M.; MOITA, G.C. Uma introdução à análise exploratória de dados multivariados. Química Nova, v.21, p.467-469, 1998. 
MOURA, M.C.S.; LOPES, A.N.C.; MOITA, G.C.; MOITA NETO, J.M. Estudo multivariado de solos surbanos da cidade de Teresina. Química Nova, v.29, p.429-435, 2006.

NASCIMENTO, C.W.A.; FONTES, R.L.F.; NEVES, J.C.L.; MELÍCIO, A.C.F.D. Fracionamento, dessorção e extração química de zinco em Latossolos. Revista Brasileira de Ciência do Solo, v.26, p.599- 606, 2002.

ORTIZ, F.R.; RODRIGUES, B.O.; BORKERT, C.M. Extratores para quantificação de cobre, zinco e manganês em solos arenosos. Ciências Agrárias, v.8, p.95-98, 2007.

SHUMAN, L.M. Chemical forms of micronutrients in soils. In: MORTVEDT, J.J.; COX, F.R.;SHUMAN, L.M.; WELCH, R.M. (Ed.). Micronutrients in agriculture. 2.ed. Madison: Soil Science Society American, 1991. p.113- 144.

UDO, E.J.; FAGBAMI, A.A. The profile distribution of total and extractable zinc in selected Nigerian soils. Communications in Soil Science and Plant Analysis, V.10, p.1141-1161, 1979.

VALADARES, J.M.A.S. O zinco em solos do estado de São Paulo. 1972. 72p. Tese (Doutorado em Solos e Nutrição de Plantas) - Universidade de São Paulo ESALQ, Piracicaba.

VALLADARES, G.S.; CAMARGO, A.O.; CARVALHO, J.R.P.; SILVA, A.M.C. Assessment of heavy metals in soils of a vineyard region with the use of principal component analysis. Scientia Agricola, v.66, p.361-367, 2009.

VALLADARES, G.S.; GOMES, E.G.; MELLO, J.C.C.B.S.; PEREIRA, M.G.; ANJOS, L.H.C.; EBELING, A.G.; BENITES, V.M. Análise dos componentes principais e métodos multicritério ordinais no estudo de organossolos e solos afins. Revista Brasileira de Ciência do Solo, v.32, p.285-296, 2008.

VALLADARES, G.S.; PEREIRA, M.G.;SOUZA, J.M.P.F.; PÉREZ, D.V.; ANJOS, L.H.C. Teores de cobre, zinco, ferro e manganês em amostras de horizontes superficiais de solos do Estado do Rio de Janeiro e suas correlações com o pH e o teor de argila.. In: CONGRESSO BRASILEIRO DE CIÊNCIA DO SOLO, 25. 1997, Rio de Janeiro. Resumos. Rio de Janeiro: Sociedade Brasileira de Ciência do Solo, 1997. CD-ROM.

VIRO, P.J. Use of ethylenediaminetetraacetic acid in soil analysis. I. Experimental. Soil Science, v.79, p.459-465, 1955.

WEAR, J.I.; SOMMER, A.L. Acid-extractable zinc of soils in relation to the occurrence of zinc deficiency symptoms of corn: a method analysis. Soil Science American Proceendings, v.12, p.143-144, 1948. 